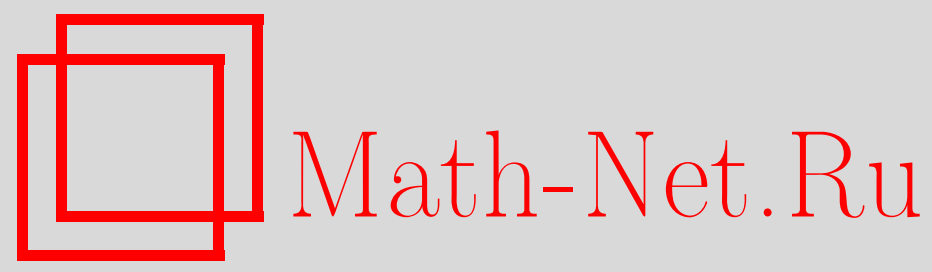

А. Н. Панов, Расширения Оре алгебр Хопфа, Матем. заметки, 2003, том 74, выпуск 3, 425-434

DOI: https://doi.org/10.4213/mzm276

Использование Общероссийского математического портала Math-Net.Ru подразумевает, что вы прочитали и согласны с пользовательским соглашением http://www . mathnet.ru/rus/agreement

Параметры загрузки:

IP : 54.210 .77 .194

26 апреля 2023 г., 12:17:00

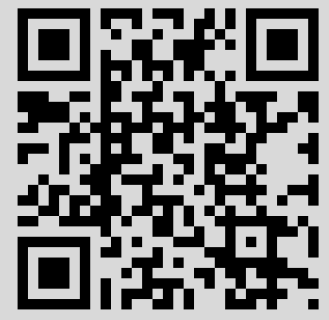


УДК 512.5

\section{РАСШИРЕНИЯ ОРЕ АЛГЕБР ХОПФА}

\section{А. Н. Панов}

В работе изучаются расширения Оре в классе алгебр Хопфа. Классификационная теорема позволяет описать расширения Хопфа-Оре для групповых алгебр, $U(\mathfrak{g})$, $U_{q}(\mathfrak{g})$, квантовой “ $a x+b$ ”.

Библиография: 8 названий.

Расширение Оре- это хорошо известная конструкция расширения колец, применимая к некоммутативньп кольцам. В результате получается кольцо, которое часто назьвают кольцом косых многочленов над заданньм кольцом. Во многих работах исследовались идеалы в этих расширениях, представления, структура центра и другие вопросы. В последних работах [1]-[5] с помошью итеративных расширений Оре были построены новые примеры алгебр Хопфа (часто конечной размерности). Цель этой работы изучить расширения Оре алгебр Хопфа в общей постановке.

1. Основная теорема. В этом разделе мы докажем критерий для расширения Оре алгебры Хопфа, которая является алгеброй Хопфа с коумножением (1.3). В следующем разделе мы используем этот критерий для классификации расширений Хопфа-Оре.

Пусть $k$ - коммутативное кольцо с 1 и $A-k$-алгебра. Рассмотрим эндоморфизм $\tau$ алгебры $A$ над $k$ и $\tau$-дифференцирование $\delta$ алгебры $A$. То есть $\delta(k)=0$ и

$$
\delta(a b)=\delta(a) b+\tau(a) \delta(b) .
$$

Расширение Ope $R=A[y ; \tau, \delta] \quad k$-алгебры $A$ есть $k$-алгебра $R$, порожденная переменной $y$ и $A$ с соотношением

$$
y a=\tau(a) y+\delta(a)
$$

для всех $a \in A$. Каждый элемент в $R=A[y ; \tau, \delta]$ единственным образом представляется в виде $\sum c_{i} y^{i}[6,2.1]$.

ОПрЕДЕЛЕНИЕ 1.0. Пусть $A$ и $R=A[y ; \tau, \delta]-$ алгебры Хопфа над $k$. Назовем $R=$ $A[y ; \tau, \delta]$ расширением Хопфа-Оре, если $\Delta(y)=y \otimes r_{1}+r_{2} \otimes y$ для некоторых $r_{1}, r_{2} \in A$ и $A$ - подалгебра Хопфа в $R$.

В дальнейшем будем использовать общее обозначение для коумножения $\Delta$, коединищы $\varepsilon$ и антипода $S$ для алгебр $A$ и $R$. Будем использовать обозначения Свидлера

Работа выполнена при поддержке Российского фонда фундаментальных исследований, грант № 02-01-00017. 
$\Delta y=a_{1} \otimes a_{2}$ (подразумевается суммирование) для коумножения. Свойство коассоциативности коумножения будет записываться как $a_{11} \otimes a_{12} \otimes a_{2}=a_{1} \otimes a_{21} \otimes a_{22}$. Элемент, определенньй последним равенством, будем обозначать $a_{1} \otimes a_{2} \otimes a_{3}$.

Заметим, что элементы $r_{1}, r_{2}$ в определении 1.1 групповые. Действительно, сравнивая $(1 \otimes \Delta) \Delta y=y \otimes \Delta\left(r_{1}\right)+r_{2} \otimes y \otimes r_{1}+r_{2} \otimes r_{2} \otimes y$ и $(\Delta \otimes 1) \Delta y=y \otimes r_{1} \otimes r_{1}+r_{2} \otimes y \otimes r_{1}$ $+\Delta\left(r_{2}\right) \otimes y$, получаем $\Delta r_{i}=r_{i} \otimes r_{i}$.

Заменим образующий элемент $y$ на $y^{\prime}=y r_{1}^{-1}$ и получим $\Delta\left(y^{\prime}\right)=y^{\prime} \otimes 1+r \otimes y^{\prime}$. Сохраняя предыдушие обозначения, мы предположим в дальнейшем, что $y$ в расширении Хопфа-Оре удовлетворяет

$$
\Delta(y)=y \otimes 1+r \otimes y
$$

с некоторым групповым элементом $r \in A$. Как обычно, $\operatorname{Ad}_{r}(a)=r a S(r)=\operatorname{rar}^{-1}$.

ЛЕмма 1.1. Если $R=A[y ; \tau, \delta]-$ расширение Хопфа-Oре, то

$$
\begin{aligned}
\varepsilon(y) & =0, \\
S(y) & =-r^{-1} y,
\end{aligned}
$$

əде $r^{-1}=S(r)$

ДокАЗАТЕЛЬСТВо. 1) Используя основное свойство коединицы $a=a_{1} \varepsilon\left(a_{2}\right)$, получаем $y=\varepsilon(y)+\varepsilon(r) y=\varepsilon(y)+y$. Это влечет (1.4).

2) С помощью основного свойства антипода, $\varepsilon(a)=a_{1} S\left(a_{2}\right)$, получаем $0=\varepsilon(y)=y+$ $r S(y)$ и $(1.5)$.

Лемма 1.2. Если $\Delta(b)=b \otimes 1$, mo $b \in k \subset A$.

ДокАЗАТЕЛЬСТВО. Используя основное свойство коединицы $a=a_{1} \varepsilon\left(a_{2}\right)=\varepsilon\left(a_{1}\right) a_{2}$, имеем $b=\varepsilon(b) \in k$.

Tеорема 1.3. $R=A[y ; \tau, \delta]$ есть расиирение Хопфа-Оре тогда и только тогда, $\kappa о г \partial a$

1) существует характер $\chi: A \mapsto k$ такой, что

$$
\tau(a)=\chi\left(a_{1}\right) a_{2}
$$

для всех $a \in A$ (то есть $\tau$ винтовой автоморфизм $A)$;

2) имеет место соотношение

$$
\chi\left(a_{1}\right) a_{2}=\operatorname{Ad}_{r}\left(a_{1}\right) \chi\left(a_{2}\right)
$$

3) $\tau$-дифферениирование $\delta$ удовлетворяет

$$
\Delta \delta(a)=\delta\left(a_{1}\right) \otimes a_{2}+r a_{1} \otimes \delta\left(a_{2}\right) .
$$


ДокАЗАТЕльство разбивается на три шага. В шаге 1 мы показываем, что коумножение $\Delta$ продолжается с $A$ на $R=A[y ; \tau, \delta]$ через (1.3) тогда и только тогда, когда имеют место (1.6)-(1.8). В шаге 2 мы доказьваем, что $R$ (с указанным вьше коумножением) допускает продолжение коединицы с $A$ через (1.4). В шаге 3 мы показьваем, что $R$ (с указанным вьше коумножением и коединищей) имеет антипод $S$, продолжающий антипод $\left.S\right|_{A}$ через (1.5).

Шаг 1. Коумножение. Предположим, что коумножение $\left.\Delta\right|_{A}$ может быть продолжено на $R=A[y ; \tau, \delta]$ через (1.3). Тогда гомоморфизм $\Delta$ сохраняет (1.2). То есть $\Delta y \Delta a=$ $\Delta \tau(a) \Delta y+\Delta \delta(a)$. Мы имеем

$$
\begin{gathered}
\Delta y \Delta a=(y \otimes 1+r \otimes y) a_{1} \otimes a_{2}=\left(\tau\left(a_{1}\right) y+\delta\left(a_{1}\right)\right) \otimes a_{2}+r a_{1} \otimes\left(\tau\left(a_{2}\right) y+\delta\left(a_{2}\right)\right) \\
=\left(\tau\left(a_{1}\right) \otimes a_{2}\right)(y \otimes 1)+\left(r a_{1} r^{-1} \otimes \tau\left(a_{2}\right)\right)(r \otimes y)+\delta\left(a_{1}\right) \otimes a_{2}+r a_{1} \otimes \delta\left(a_{2}\right), \\
\begin{aligned}
\Delta \tau(a) \Delta y+\Delta \delta(a) & =\Delta \tau(a)(y \otimes 1+r \otimes y)+\Delta \delta(a) \\
& =\Delta \tau(a) y \otimes 1+\Delta \tau(a) r \otimes y+\Delta \delta(a) .
\end{aligned}
\end{gathered}
$$

Видно, что $\Delta$ сохраняет (1.2) тогда и только тогда, когда вьполняются следуюшие условия:

$$
\begin{aligned}
& \Delta \tau(a)=\tau\left(a_{1}\right) \otimes a_{2}, \\
& \Delta \tau(a)=\operatorname{Ad}_{r}\left(a_{1}\right) \otimes \tau\left(a_{2}\right), \\
& \Delta \delta(a)=\delta\left(a_{1}\right) \otimes a_{2}+r a_{1} \otimes \delta\left(a_{2}\right)
\end{aligned}
$$

для всех $a \in A$ и $\Delta a=a_{1} \otimes a_{2}$. Последнее условие совпадает с (1.8).

Докажем, что из (1.9) и (1.10) вытекают (1.6)-(1.7). Рассмотрим элемент $\chi(a):=$ $\tau\left(a_{1}\right) S\left(a_{2}\right) \in A$. Прямыми вычислениями получаем

$$
\begin{aligned}
\Delta \chi(a) & =\Delta \tau\left(a_{1}\right) \Delta S\left(a_{2}\right)=\left(\tau\left(a_{11}\right) \otimes a_{12}\right)\left(S\left(a_{22}\right) \otimes S\left(a_{21}\right)\right) \\
& =\tau\left(a_{11}\right) S\left(a_{22}\right) \otimes a_{12} S\left(a_{21}\right)=\tau\left(a_{1}\right) S\left(a_{4}\right) \otimes\left(a_{2} S\left(a_{3}\right)\right) \\
& =\tau\left(a_{1}\right) S\left(a_{3}\right) \otimes \varepsilon\left(a_{2}\right)=\tau\left(a_{1} \varepsilon\left(a_{2}\right)\right) S\left(a_{3}\right) \otimes 1=\tau\left(a_{1}\right) S\left(a_{2}\right) \otimes 1=\chi(a) \otimes 1 .
\end{aligned}
$$

Из леммы 1.2 имеем $\chi(a) \in k$. Можно рассматривать $\chi$ как отображение $\chi: A \rightarrow k$. Так как $\tau$ эндоморфизм, а $\chi$ характер, то

$$
\begin{aligned}
\chi(a b) & =\tau\left(a_{1} b_{1}\right) S\left(a_{2} b_{2}\right)=\tau\left(a_{1}\right) \tau\left(b_{1}\right) S\left(b_{2}\right) S\left(a_{2}\right)=\tau\left(a_{1}\right) \chi(b) S\left(a_{2}\right)=\chi(a) \chi(b), \\
\chi(a+b) & =\tau\left((a+b)_{1}\right) S\left((a+b)_{2}\right)=\tau\left(a_{1}\right) S\left(a_{2}\right)+\tau\left(b_{1}\right) S\left(b_{2}\right)=\chi(a)+\chi(b) .
\end{aligned}
$$

Через $\chi$ можно восстановить $\tau$ :

$$
\chi\left(a_{1}\right) a_{2}=\tau\left(a_{1}\right) S\left(a_{2}\right) a_{3}=\tau\left(a_{1}\right) \varepsilon\left(a_{2}\right)=\tau\left(a_{1} \varepsilon\left(a_{2}\right)\right)=\tau(a) .
$$

Это доказьвает (1.6). Подставляем $\tau(a)$ в (1.10) и получаем

$$
\begin{gathered}
\Delta\left(\chi\left(a_{1}\right) a_{2}\right)=\operatorname{Ad}_{r}\left(a_{1}\right) \otimes \chi\left(a_{2}\right) a_{3}, \quad \chi\left(a_{1}\right) \Delta\left(a_{2}\right)=\operatorname{Ad}_{r}\left(a_{1}\right) \chi\left(a_{2}\right) \otimes a_{3}, \\
\left(\chi\left(a_{1}\right) a_{2}\right) \otimes a_{3}=\operatorname{Ad}_{r}\left(a_{1}\right) \chi\left(a_{2}\right) \otimes a_{3} .
\end{gathered}
$$




\section{Отсюда}

$$
\begin{gathered}
\left(\chi\left(a_{1}\right) a_{2}\right) a_{3} S\left(a_{4}\right)=\operatorname{Ad}_{r}\left(a_{1}\right) \chi\left(a_{2}\right) a_{3} S\left(a_{4}\right), \quad\left(\chi\left(a_{1}\right) a_{2}\right) \varepsilon\left(a_{3}\right)=\operatorname{Ad}_{r}\left(a_{1}\right) \chi\left(a_{2}\right) \varepsilon\left(a_{3}\right), \\
\chi\left(a_{1}\right)\left(a_{2}\right)=\operatorname{Ad}_{r}\left(a_{1}\right) \chi\left(a_{2}\right) .
\end{gathered}
$$

Это доказьвает (1.7). Мы доказали, что (1.6)-(1.8) являются необходимыми условиями для продолжения коумножения.

С другой стороны, если (1.6)-(1.8) имеют место, то $\Delta \tau(a)=\chi\left(a_{1}\right) a_{2} \otimes a_{3}=\tau\left(a_{1}\right) \otimes a_{2}$ и $\Delta \tau(a)=\Delta\left(\operatorname{Ad}_{r} a_{1} \chi\left(a_{2}\right)\right)=\operatorname{Ad}_{\Delta r}\left(a_{1} \otimes a_{2}\right) \chi\left(a_{3}\right)=\operatorname{Ad}_{r} a_{1} \otimes \operatorname{Ad}_{r} a_{2} \chi\left(a_{3}\right)=\operatorname{Ad}_{r} a_{1} \otimes$ $\tau\left(a_{2}\right)$. Это доказьвает, что верны (1.9) и (1.10) и коумножение $\left.\Delta\right|_{A}$ продолжается до гомоморфизма $\Delta: R \rightarrow R \otimes R$. Так как $(1 \otimes \Delta) \Delta(a)=(\Delta \otimes 1) \Delta(a)$ для любого $a \in A$ и $(1 \otimes \Delta) \Delta(y)=(\Delta \otimes 1) \Delta(y)$, то $(1 \otimes \Delta) \Delta=(\Delta \otimes 1) \Delta$. Отображение $\Delta: R \rightarrow R \otimes R$ является коумножением.

Шаг 2. Коединища. Пусть $R$ допускает коумножение (т.е. верны (1.6)-(1.8)). Докажем существование коединищы, продолжающей $\left.\varepsilon\right|_{A}$ и удовлетворяющей (1.4). Можно продолжить $\varepsilon$ как гомоморфизм $R$, если $\varepsilon$ сохраняет (1.2). То есть $\varepsilon(y) \varepsilon(a)=\varepsilon(\tau(a)) \varepsilon(y)$ $+\varepsilon(\delta(a))$. Так как $\varepsilon(y)=0$, то $\varepsilon$ допускаетпродолжение на $R$ тогда и только тогда, когда

$$
\varepsilon(\delta(a))=0
$$

Наша цель доказать (1.11). Действуя $m(1 \otimes \varepsilon)$ на $(1.8)$, получаем

$$
\begin{gathered}
m(\mathrm{id} \otimes \varepsilon)(\Delta \delta(a))=m(\mathrm{id} \otimes \varepsilon)\left(\delta\left(a_{1}\right) \otimes a_{2}+r a_{1} \otimes \delta\left(a_{2}\right)\right), \\
\delta(a)=\delta\left(a_{1}\right) \varepsilon\left(a_{2}\right)+r a_{1} \varepsilon\left(\delta a_{2}\right)=\delta(a)+r a_{1} \varepsilon\left(\delta a_{2}\right) .
\end{gathered}
$$

Получаем $a_{1} \varepsilon\left(\delta\left(a_{2}\right)\right)=0$. Окончательно,

$$
0=S\left(a_{1}\right) a_{2} \varepsilon\left(\delta\left(a_{3}\right)\right)=\varepsilon\left(a_{1}\right) \varepsilon\left(\delta a_{2}\right)=\varepsilon \delta\left(\varepsilon\left(a_{1}\right) a_{2}\right)=\varepsilon(\delta(a)) .
$$

Это доказывает (1.11) и существование продолжения $\varepsilon: R \rightarrow k$ через (1.4). Используя разложение $b=\sum c_{i} y^{i}$ произвольного элемента $b \in R$, легко доказьвается, что $\varepsilon: R \rightarrow$ $k$ - коединица биалгебры $R$.

Шаг 3. Антипод. Пусть $R$ как и в шаге 2 . Напомним, что $S$ является антиавтоморфизмом алгебры Хопфа и $\Delta S(a)=S\left(a_{2}\right) \otimes S\left(a_{1}\right)$ [8,1.5.10]. Если $R$ допускает антипод $S$, которьй продолжается (как антиавтоморфизм) с $A$ на $R$ через (1.5), то $S$ сохраняет (1.2). То есть

$$
S(a) S(y)=S(y) S \tau(a)+S \delta(a) .
$$

С другой стороны, если (1.12) верно, то $S$ продолжается как антиавтоморфизм с $A$ на $R$ через (1.5). Используя разложение $b=\sum c_{i} y^{i}$ произвольного элемента $b \in R$, легко видно, что определенньй $S: R \rightarrow R$ является антиподом $R$.

Следовательно, существование антипода $R$ с (1.5) равносильно (1.12). Из (1.5)

$$
\begin{aligned}
& -S(a) r^{-1} y=-r^{-1} y S \tau(a)+S \delta(a) \\
& -S(a) r^{-1} y=-r^{-1} \tau(S \tau(a)) y-r^{-1} \delta(S \tau(a))+S \delta(a) .
\end{aligned}
$$


Условия (1.12) вьполнено тогда и только тогда, когда выполнены следуюшие два условия:

$$
\begin{aligned}
S(a) r^{-1} & =r^{-1} \tau S \tau(a) \\
r S \delta(a) & =\delta S \tau(a)
\end{aligned}
$$

Докажем (1.13):

$$
\begin{aligned}
\tau S \tau(a) & =\tau S \chi\left(a_{1}\right) a_{2}=\tau\left(\chi\left(a_{1}\right) S\left(a_{2}\right)\right)=\chi\left(a_{1}\right) \tau S\left(a_{2}\right) \\
& =\chi\left(a_{1}\right) \operatorname{Ad}_{r} S\left(a_{3}\right) \chi S\left(a_{2}\right)=\chi\left(a_{1} S\left(a_{2}\right)\right) \operatorname{Ad}_{r} S\left(a_{3}\right)=\operatorname{Ad}_{r} S(a) .
\end{aligned}
$$

Следующая цель доказать (1.14). Из (1.6) получаем $S \tau(a)=S\left(\chi\left(a_{1}\right) a_{2}\right)=\chi\left(a_{1}\right) S\left(a_{2}\right)$. Представляем (1.14) в эквивалентной форме:

$$
r S \delta(a)=\chi\left(a_{1}\right) \delta S\left(a_{2}\right)
$$

Обозначим $L=r S \delta(a)$ и $M=\chi\left(a_{1}\right) \delta S\left(a_{2}\right)$. Действуя $m(\mathrm{id} \otimes S)$ на $(1.8)$, получаем

$$
\begin{gathered}
m(\mathrm{id} \otimes S)(\Delta \delta(a))=m(\mathrm{id} \otimes S)\left(\delta\left(a_{1}\right) \otimes a_{2}+r a_{1} \otimes \delta\left(a_{2}\right)\right), \\
0=\varepsilon \delta(a)=\delta\left(a_{1}\right) S\left(a_{2}\right)+r a_{1} S \delta\left(a_{2}\right), \quad a_{1} S \delta\left(a_{2}\right)=-r^{-1} \delta\left(a_{1}\right) S\left(a_{2}\right) .
\end{gathered}
$$

Тогда

$$
\begin{aligned}
L & =r S \delta(a)=r \varepsilon\left(a_{1}\right) S \delta\left(a_{2}\right)=r S\left(a_{1}\right) a_{2} S \delta\left(a_{3}\right) \\
& =-r S\left(a_{1}\right) r^{-1} \delta\left(a_{2}\right) S\left(a_{3}\right)=-\operatorname{Ad}_{r} S\left(a_{1}\right) \delta\left(a_{2}\right) S\left(a_{3}\right) .
\end{aligned}
$$

С другой стороны, действуя $\delta$ на левую и правую части $\varepsilon(a)=a_{1} S a_{2}$, имеем

$$
\begin{aligned}
0 & =\delta\left(a_{1}\right) S\left(a_{2}\right)+\tau\left(a_{1}\right) \delta S\left(a_{2}\right), \\
M & =\chi\left(a_{1}\right) \delta S\left(a_{2}\right)=\chi\left(a_{1}\right) \varepsilon\left(a_{2}\right) \delta S\left(a_{3}\right)=\chi\left(a_{1}\right) \tau\left(S\left(a_{2}\right) a_{3}\right) \delta S\left(a_{4}\right) \\
& =\chi\left(a_{1}\right) \tau S\left(a_{2}\right) \tau\left(a_{3}\right) \delta S\left(a_{4}\right)=-\chi\left(a_{1}\right) \tau S\left(a_{2}\right) \delta\left(a_{3}\right) S\left(a_{4}\right) \\
& =-\chi\left(a_{1}\right) \operatorname{Ad}_{r} S\left(a_{3}\right) \chi\left(S\left(a_{2}\right)\right) \delta\left(a_{4}\right) S\left(a_{5}\right)=-\operatorname{Ad}_{r} S\left(a_{1}\right) \delta\left(a_{2}\right) S\left(a_{3}\right) .
\end{aligned}
$$

Сравнивая (1.17) и (1.16), заключаем $L=M$. Это доказьвает (1.15) и существование антипода.

СлЕдСТВИЕ 1.4. Если $R=A[y ; \tau, \delta]$ - расширение Хопфа-Оре и $A$ - кокоммутативная алгебра Хопфа, то $r$ лежит в иентре $A$.

ДокАЗАТЕЛЬСТВО. Поскольку $\tau(a)=\chi\left(a_{1}\right)\left(a_{2}\right)=\operatorname{Ad}_{r}\left(a_{1}\right) \chi\left(a_{2}\right)=\chi\left(a_{1}\right) \operatorname{Ad}_{r} a_{2}$, TO

$$
\begin{gathered}
\chi\left(S\left(a_{1}\right)\right) \chi\left(a_{2}\right) a_{3}=\chi\left(S\left(a_{1}\right)\right) \chi\left(a_{2}\right) \operatorname{Ad}_{r} a_{3}, \\
\chi\left(\varepsilon\left(a_{1}\right)\right) a_{2}=\chi\left(\varepsilon\left(a_{1}\right)\right) \operatorname{Ad}_{r} a_{2}, \quad a=\operatorname{Ad}_{r}(a)
\end{gathered}
$$

для всех $a \in A$. 
ЗАмечАниЕ 1.5. 1) Условие (1.6) заключает, что $\tau(a)=\chi\left(a_{1}\right) a_{2}$. То есть $\tau$-правьй $\chi$-винтовой автоморфизм $R[7,1.6 .5]$. Автоморфизм $\tau^{\prime}(a)=a_{1} \chi\left(a_{2}\right)$ назьвается левым $\chi$-винтовым автоморфизмом. Условие (1.7) может быть переписано в эквивалентной форме $\tau=\operatorname{Ad}_{r} \tau^{\prime}$.

2) Обозначим через $\mathscr{R}_{r}$ и $\mathscr{L}_{r}$ правое и левое винтовое действие $r$ в $A^{*}$. По определению $\mathscr{R}_{r} \lambda(a)=\lambda(r a)$ и $\mathscr{L}_{r} \lambda(a)=\lambda(a r)$ для всех $\lambda \in A^{*}$ и $a \in A$. Условие (1.7) имеет эквивалентную форму $\mathscr{R}_{r}=\operatorname{Ad}_{\chi} \mathscr{L}_{r}$.

ОПрЕДЕлЕниЕ 1.6. 1) Отображение $\delta$, удовлетворяющее (1.8), будем назьвать $r$-кодифферениированием (заметим, что $\delta$ - $r$-кодифференцирование, если сопряженное отображение $\delta^{*}: A^{*} \rightarrow A^{*}$ является $\mathscr{R}_{r}$-дифференшированием);

2 ) будем называть $\delta-\langle\chi, r\rangle$-дифференцированием, если $\delta-\tau$-дифференцирование (см. 1.1) и $r$-кодифференцирование.

ОБозначение 1.7. Расширение Хопфа-Оре $R=A[y ; \tau, \delta]$ будем обозначать $R=$ $A(\chi, r, \delta)$, где $\chi: A \rightarrow k$-характер, $r$ - групповой элемент $A$, удовлетворяющий (1.7), $\delta-\langle\chi, r\rangle$-дифференцирование.

ОПРЕДЕЛЕНИЕ 1.8. Назовем $\delta$ внутренним $\langle\chi, r\rangle$-дифферениированием, если существует элемент $d \in A$ такой, что $\delta(a)=\tau(a) d-d a$ и $\Delta(d)=d \otimes 1+r \otimes d$.

ОпредЕлЕниЕ 1.9. Назовем два расширения Хопфа-Оре $R$ и $R^{\prime}$ алгебр Хопфа $A$ и $A^{\prime}$ изоморфными, если существует изоморфизм алгебр Хопфа $\Phi: R \mapsto R^{\prime}$, переводящий $A$ на $A^{\prime}$.

ПРЕДЛОЖЕНИЕ 1.10. 1) Если $\delta$ - внутреннее $\langle\chi, r\rangle$-дифферениирование, то $A(\chi$, $r, \delta)$ изоморфно $A(\chi, r, 0)$.

2) Два расширения Хопфа-Оре $R=A(\chi, r, \delta)$ u $R=A^{\prime}\left(\chi^{\prime}, r^{\prime}, \delta^{\prime}\right)$ изоморфны, если существует изоморфизм алгебр Хопфа $\Phi: A \mapsto A^{\prime}$ такой, что $\chi^{\prime}=\chi^{-1}$, $r^{\prime}=\Phi(r), \delta^{\prime}=\Phi \delta \Phi^{-1}+\delta^{\prime \prime}$, где $\delta^{\prime \prime}-$ внутреннее $\left\langle\chi^{\prime}, r^{\prime}\right\rangle$-дифференцирование $R^{\prime}$.

ДокАЗАТЕЛЬСтво. Замена $y$ на $y+d$.

ПРЕДЛОЖЕНИЕ 1.11. Подалгебра Хопфа $A$ в $R=A(\chi, r, \delta)$ является нормальной (см. [7, 3.4.1]) тогда и только тогда, когда $\chi=\varepsilon$ и гежит в иентре $A$.

ДокАЗАтЕльство. Левое (соответственно правое) присоединенное действие алгебры Хопфа $H$ определяется как $\left(\operatorname{ad}_{l} h\right)(x)=h_{1} x S\left(h_{2}\right)\left(\right.$ соответственно $\left(\operatorname{ad}_{r} h\right)(x)=$ $\left.S\left(h_{1}\right) x h_{2}\right)$. Подалгебра Хопфа называется нормальной, если она инвариантна относительно левого и правого присоединенного действия. Подалгебра Хопфа $A$ в $R=$ $A(\chi, r, \delta)$ является нормальной, если $\left(\operatorname{ad}_{l} y\right)(a) \in A$ и $\left(\operatorname{ad}_{r} y\right)(a) \in A$ для любого $a \in A$. То есть $y a-r a r^{-1} y \in A$ и $-r^{-1} y a+r^{-1} a y=r^{-1}(-y a+a y) \in A$. Из (1.2) вытекает, что $\tau(a)=r a r^{-1}$ и $\tau(a)=a$ для любого $a \in A$. Это доказьвает утверждение.

2. Классификация расширений Хопфа-Оре. В этом разделе мы проведем классификацию расширений Хопфа-Оре для некоторьх классов алгебр Хопфа.

Пункт 1. Кокоммутативные алгебры Хопфа. Здесь мы представляем метод построения $\langle\chi, r\rangle$-дифференцирований кокоммутативных алгебр Хопфа.

Пусть $A$ - кокоммутативная алгебра Хопфа. Пусть $\chi$ - характер и $r$-групповой элемент из центра $A$. Пусть $\alpha$ - линейная форма $\alpha: A \rightarrow k$, удовлетворяющая

$$
\alpha(u v)=\alpha(u) \varepsilon(v)+\chi(u) \alpha(v) .
$$


ПРЕДЛОЖЕНИЕ 2.1. Отображсение

$$
\delta(a):=\alpha\left(a_{1}\right)(1-r) a_{2}
$$

является $\langle\chi, r\rangle$-дифференцированием. Это позволяет построить пример $A(\chi, \tau, \delta)$ из расширения Хопфа-Оре $A$.

ДокАЗАТЕльСтво. Отображение $\delta$ является $\tau$-дифференцированием:

$$
\begin{aligned}
\delta(a b) & =\alpha\left((a b)_{1}\right)(1-r)(a b)_{2}=\left(\alpha\left(a_{1}\right) \varepsilon\left(b_{1}\right)+\chi\left(a_{1}\right) \alpha\left(b_{1}\right)\right)(1-r) a_{2} b_{2} \\
& =\alpha\left(a_{1}\right)(1-r) a_{2} \varepsilon\left(b_{1}\right) b_{2}+\chi\left(a_{1}\right) a_{2} \alpha\left(b_{1}\right)(1-r) b_{2}=\delta(a) b+\tau(a) \delta(b) .
\end{aligned}
$$

Отображение $\delta$ является $r$-кодифференцированием (см. 1.8):

$$
\begin{aligned}
\Delta \delta(a) & =\alpha\left(a_{1}\right) \Delta(1-r) a_{2}=\alpha\left(a_{1}\right)(1 \otimes 1-r \otimes r) a_{2} \otimes a_{3} \\
& =\alpha\left(a_{1}\right)(1-r) a_{2} \otimes a_{3}+\alpha\left(a_{1}\right) r a_{2} \otimes(1-r) a_{3} \\
& =\alpha\left(a_{1}\right)(1-r) a_{2} \otimes a_{3}+r a_{1} \otimes \alpha\left(a_{2}\right)(1-r) a_{3}=\delta\left(a_{1}\right) \otimes a_{2}+r a_{1} \otimes \delta\left(a_{2}\right) .
\end{aligned}
$$

ЗАмечаниЕ. В случае $A=k G$ линейная форма $\alpha$ определяется $\alpha(g), g \in G$, и удовлетворяет $\alpha(g h)=\alpha(g)+\chi(g) \alpha(h)$. То есть $\alpha$ есть 1-коцикл $\left(\alpha \in Z_{\chi}^{1}(G)\right)$.

Пункт 2. Групшовые алгебры.

ПРЕДЛОЖЕНИЕ 2.2. Каждое расширение Хопфа-Оре для $A=k G$ имеет вид $A(\chi, r, \delta)$, где $\chi$ - характер группы, $r$ - әлемент иентра группы $G$ и $\delta$ имет вид (2.2) для некоторого $\alpha \in Z_{\chi}^{1}(G)$.

ДокАЗАТЕЛЬСтво. Из теоремы 1.3 все расширения Хопфа-Оре имеют вид $A(\chi, r, \delta)$, где $\chi-\operatorname{xapaктер~} k G$ (т.е. $\chi$ характер группы $G$ ), $r$ - групповой элемент в $k G$ (все групповые элементы $k G$ принадлежат $G)$ и $\delta$ является $\langle\chi, r\rangle$-дифференцированием. Было доказано, что $r$ содержится в центре $G$ (следствие 1.4). Условие (1.8) имеет вид

$$
\Delta \delta(g)=\delta(g) \otimes g+r g \otimes \delta(g)
$$

для всех $g \in G$. Элемент $c:=\delta(g) g^{-1}$ удовлетворяет

$$
\Delta(c)=c \otimes 1+r \otimes c
$$

Для любого $\mu \in(k G) *$ имеет место

$$
\mu^{2}(c)=\mu \otimes \mu(\Delta c)=\mu(c) \mu(1)+\mu(r) \mu(c)
$$

Элемент $c$ представим как $c=\sum \gamma(g) g$. Пусть $g_{0}$ - элемент группы $G$, отличньй от 1 и $r$. Выберем $\mu_{0} \in(k G)^{*}$ в $(2.5)$ так, что $\mu_{0}\left(g_{0}\right)=1$ и $\mu_{0}(g)=0$ для всех элементов из $G$ (в частности, $\mu_{0}(1)=\mu_{0}(r)=0$ ). Положим $\mu=\mu_{0}$ в $(2.5)$ и получим

$$
\gamma^{2}\left(g_{0}\right)=\sum \gamma(g) \mu_{0}^{2}(g)=\mu_{0}^{2}\left(\sum \gamma(g) g\right)=\mu_{0}^{2}(c)=\mu_{0}(c) \mu_{0}(1)+\mu_{0}(r) \mu_{0}(c)=0
$$


Это влечет $\gamma\left(g_{0}\right)=0$ и $c=\gamma(1)+\gamma(r) r$. Подставляя в $(2.4)$, получаем $c=\gamma(1-r)$ для некоторого $\gamma \in k$. Следовательно, $\delta(g)=\alpha(g)(1-r) g$ для некоторого $\alpha: G \rightarrow k$. Пусть $r \neq 0$. Так как $\delta(g)-\tau$-дифференцирование, то $\delta(g h)=\delta(g) h+\chi(g) \delta(h)$. После подстановки $\delta(g)=\alpha(g)(1-r) g$ получаем $(1-r)(\alpha(g h)-\alpha(g)-\chi(g) \alpha(h)) g h=0$. Окончательно, $\alpha(g) \in Z \chi^{1}(G)$.

Пункт 3. $A=U(\mathfrak{g})$, где $\mathfrak{g}$ - алгебра Ли над полем $k$ нулевой характеристики. Из $[8,2.7 .2]$ легко вытекает, что любой групповой элемент в $U(\mathfrak{g})$ равен 1 . Имеем $r=1$. Характер $\chi$ алгебры $U(\mathfrak{g})$ определяется $\mathfrak{g}$-характером и $\tau(x)=x+\chi(x)$ для всех $x \in \mathfrak{g}$. Для $x \in \mathfrak{g}$ мы имеем $\Delta(x)=x \otimes 1+1 \otimes x$ и $(1.8)$ имеет вид $\Delta \delta(x)=\delta(x) \otimes 1+1 \otimes \delta(x)$ (т.е. $\delta(x)$ примитивньй элемент в $U(\mathfrak{g}))$. Из PBW-теоремы в случае нулевой характеристики все примитивные элементы лежат в $\mathfrak{g}$. Следовательно, $\delta(x) \in \mathfrak{g}$ и $\delta$ отображает $\mathfrak{g}$ в $\mathfrak{g}$. Отображение удовлетворяет условию

$$
\delta\left(\left[x_{1}, x_{2}\right]\right)=\left[\delta\left(x_{1}\right), x_{2}\right]+\left[x_{1}, \delta\left(x_{2}\right)\right]+\chi\left(x_{1}\right) \delta\left(x_{2}\right)-\chi\left(x_{2}\right) \delta\left(x_{2}\right) .
$$

Мы доказали

ПРЕДЛОЖЕНИЕ 2.3. Любое расширение Хопфа-Оре $R$ алгебры Хопфа $U(\mathfrak{g})$ порождается у и $U(\mathfrak{g})$ с условием $y x=(x+\chi(x)) y+\delta(x)$ для некоторого характера ұ алгебры Ли $\mathfrak{g}$ и линейного отображсеня $\delta: \mathfrak{g} \rightarrow \mathfrak{g}$, удовлетворяющего (2.6). $B$ случае $\chi=0$ алгебра $R$ является универсальной обертывающей алгеброй $U\left(\mathfrak{g}^{\prime}\right)$ алгебры $Л и \mathfrak{g}^{\prime}=k y+\mathfrak{g}$.

ПримеР 2.4. Предложение неверно в случае поля положительной характеристики. Пусть $k$ - поле характеристики $p>0$ и $U=k[x]$ - универсальная обертьвающая алгебра для $\mathfrak{g}=k x$. Полагая $r=1, \tau(x)=x$ и $\delta(x)=x^{p}$, получаем расширение Хопфа-Оре, порожденное $y, x$, с условием $y x=x y+x^{p}$.

Пункт 4. Пусть $A$ - квантовая универсальная обертывающая алгебра $U_{q}(\mathfrak{g})$ в смысле Дринфельда-Джимбо и $q$ - переменная. Эта алгебра порождается $K_{i}, e_{i}, f_{i}, i=$ $1, \ldots, n$, с обычными соотношениями и $\Delta K_{i}=K_{i} \otimes K_{i}, \Delta e_{i}=e_{i} \otimes 1+K_{i} \otimes e_{i}$, $\Delta f_{i}=f_{i} \otimes K_{i}^{-1}+1 \otimes f_{i}$. Пусть $\left\{\alpha_{1}, \ldots, \alpha_{n}\right\}$ - система простых корней $\mathfrak{g}$.

ПРЕДЛОЖЕНИЕ 2.5. Любое расширение Хопфа-Оре для $A=U(\mathfrak{g})$ изоморфно $A(1,1, \delta)$ с $\delta\left(K_{i}\right)=0, \delta\left(e_{i}\right)=\xi_{i} e_{i} u \delta\left(f_{i}\right)=-\xi_{i} f_{i}$ для некоторых $\xi_{1}, \ldots, \xi_{n} \in k$.

ДокАЗАТЕЛьСтво. Любой характер $A$ имеет вид

$$
\chi\left(K_{i}\right)=\varepsilon_{i}= \pm 1, \quad \chi\left(e_{i}\right)=\chi\left(f_{i}\right)=0 .
$$

Тогда $\tau\left(K_{i}\right)=\varepsilon_{i} K_{i}, \tau\left(e_{i}\right)=\varepsilon_{i} e_{i}, \tau\left(f_{i}\right)=f_{i}$. Образующий элемент $y$ в расширении Хопфа-Оре алгебры $U_{q}(\mathfrak{g})$ удовлетворяет (1.3) с $r=K_{1}^{\gamma_{1}} \cdots K_{n}^{\gamma_{n}}=K^{\gamma}$. Из (1.7) вытекает $\varepsilon_{i} e_{i}=\operatorname{Ad}_{r}\left(e_{i}\right)=q^{\left(\gamma, \alpha_{i}\right)} e_{i}$ и $f_{i}=q^{-\left(\gamma, \alpha_{i}\right)} \varepsilon_{i}^{-1} f_{i}$. Здесь $\gamma=0$ и $\varepsilon_{i}=1$ для всех $i$. Отсюда $r=1$ и $\tau=\mathrm{id}$. Получаем, в частности, что элемент $y$ примитивньй. Из (1.8) $\Delta \delta(K)=\delta(K) \otimes K+K \otimes \delta(K)$. То есть $\delta(k) K^{-1}$ - примитивный элемент. Из $\mathrm{PBW}$-теоремы легко получить, что $U_{q}(\mathfrak{g})$ не имеет примитивных элементов. Это влечет $\delta(K)=0$. Имеем $y e_{i}=e_{i} y+\delta\left(e_{i}\right), y f_{i}=f_{i} y+\delta\left(f_{i}\right), y K_{i}=K_{i} y$. Из (1.8) получаем $\Delta \delta\left(e_{i}\right)=\delta\left(e_{i}\right) \otimes 1+K_{i} \otimes \delta\left(e_{i}\right)$ и $\Delta \delta\left(f_{i}\right)=\delta\left(f_{i}\right) \otimes K_{i}^{-1}+1 \otimes \delta\left(f_{i}\right)$. Отсюда $\delta\left(e_{i}\right)=\xi_{i} e_{i}$, $\delta\left(f_{i}\right)=-\xi_{i} f_{i}$ для некоторых чисел $\xi_{i}$. 
Пункт 5. Расширения Хопфа-Оре для квантовой группы “ $a x+b$ ”. Пусть $q-$ переменная. Квантовая группа " $a x+b$ " порождается над кольцом $C=k\left[q, q^{-1}\right]$ элементами $K$, $x$ с соотношениями $K x=q^{2} x K, \Delta(K)=K \otimes K, \Delta(x)=x \otimes 1+K \otimes x, \varepsilon(K)=1$, $\varepsilon(x)=0, S(K)=K^{-1}$ и $S(x)=-K^{-1} x$.

ПРЕДЛОЖЕНИЕ 2.6. Существует ровно четыре расширения Хопфа-Оре для квантовой группы “аx $+b$ ": $y K=K y, y x=x y+\gamma x$ c $\gamma=0,1, y-$ nримитивный әлемент и $K y=q^{-2} y K, y x=x y+\gamma\left(K-K^{-1}\right)$ с $\gamma=0,1, \Delta y=y \otimes K^{-1}+1 \otimes y$. Среди расширений квантовая алгебра $s l_{q}(2)$.

ДокАЗАтЕльство. Любой характер $\chi$ алгебры $A$ имеет вид $\chi(K)=\alpha=C^{*}$ и $\chi(x)=0$. Тогда $\tau(K)=\chi(K) K=\alpha K$ и $\tau(x)=\chi(K) x=\alpha x$. Элемент $r$ групповой. Отсюда $r=K^{m}$ для некоторого $m \in \mathbb{Z}$ и

$$
\Delta y=y \otimes 1+K^{m} \otimes y .
$$

Условие (1.7) имеет вид

$$
\tau(K)=\operatorname{Ad}_{K^{m}}(K) \chi(K)=\alpha K, \quad \tau(x)=\operatorname{Ad}_{K^{m}}(x) \chi(1)=q^{2 m} x .
$$

Отсюда

$$
\alpha=q^{2 m} .
$$

Условие (1.8) имеет вид $\Delta \delta(K)=\delta(K) \otimes K+K^{m+1} \otimes \delta(K)$. Элемент $b:=\delta(K) K^{-1}$ удовлетворяет уравнению

$$
\Delta b=b \otimes 1+K^{m} \otimes b .
$$

Можно показать, что любое решение (2.9) имеет вид $b=\gamma x+\gamma^{\prime}(1-K)$ (в случае $m=1)$ или $b=\gamma\left(1-K^{m}\right)$. Ниже мы покажем, что, заменяя образующий элемент $y$, можно считать $\delta(K)=0$.

1) Пусть $b=\gamma x+\gamma^{\prime}(1-K)$ и $m=1$. Тогда $\delta(K)=\gamma x K+\gamma^{\prime}(1-K) K$ и $\alpha=q^{2}$. Рассмотрим внутренние дифференцирования $\delta_{x}(a)=\tau(a) x-x a$ и $\delta_{1-K}(a)=\tau(a)(1-K)-$ $(1-K) a\left(\right.$ см. определение 1.7). Тогда $\delta_{x}(K)=\tau(K) x-x K=x K\left(\alpha q^{2}-1\right)=x K\left(q^{4}-1\right)$ и $\delta_{1-K}(K)=\left(q^{2}-1\right)(1-K) K$. Заменяя $y$ на $y^{\prime}=y+\xi x+\xi^{\prime}(1-K)$ с $\xi=\gamma\left(q^{4}-1\right)^{-1}$ и $\xi^{\prime}=\gamma^{\prime}\left(q^{2}-1\right)^{-1}$, мы получаем, что новьй образующий элемент $y^{\prime}$ удовлетворяет $(2.7)$ c $\delta^{\prime}(K)=0$.

2) Пусть $b=\gamma\left(1-K^{m}\right)$. Тогда $\delta(K)=\gamma\left(1-K^{m}\right) K$. Элемент $b$ удовлетворяет (2.9). Рассмотрим внутреннее дифференцирование $\delta_{b}$. Тогда

$$
\delta_{b}(K)=\tau(K) b-b K=\gamma(\alpha-1)\left(1-K^{m}\right) K .
$$

Если $\alpha \neq 1$, то заменим $y$ на $y^{\prime}=y+\xi b$ с $\xi=(\alpha-1)^{-1}$ и получим $\delta^{\prime}(K)=0$. Если $\alpha=1$, то уравнение $(2.8)$ дает $m=0$ и $\delta(K)=0$.

Мы доказали, что, заменяя $y$, можно считать $\delta(K)=0$. Отсюда $y K=\alpha K y$. Наша следующая цель найти $\delta(x)$. Действуя $\delta$ на соотношение $K x=q^{2} x K$, получаем $q^{2 m} K \delta(x)=q^{2} \delta(x) K$ и

$$
K \delta(x)=q^{2(1-m)} \delta(x) K .
$$

Уравнение (1.8) дает

$$
\Delta \delta(x)=\delta(x) \otimes 1+r K \otimes \delta(x)=\delta(x) \otimes 1+K^{m+1} \otimes \delta(x) .
$$


Снова существуют только два решения

$$
\delta(x)= \begin{cases}\gamma x+\gamma^{\prime}(1-K) & \text { для } m=0, \\ \gamma\left(1-K^{m+1}\right) & \text { для } m \neq 0 .\end{cases}
$$

Из (2.10) и (2.11) получаем

$$
\delta(x)= \begin{cases}\gamma x & \text { для } m=0, \\ \gamma\left(1-K^{2}\right) & \text { для } m=1 .\end{cases}
$$

В первом случае расширение Хопфа-Оре $R$ порождается $y$ и $A$ с $y K=K y, y x=x(y+\gamma)$ и $y-$ примитивный элемент. Можно считать, что $\gamma=0$ или $\gamma=1$.

Во втором случае $\delta(x)=\gamma\left(1-K^{2}\right), m=1$ и $\alpha=q^{2}$. Получаем $y K=q^{2} K y$, $y x=q^{2} x y+\gamma\left(1-K^{2}\right)$.

После замены $y^{\prime}=y K^{-1}$ имеем $\Delta y^{\prime}=y^{\prime} \otimes K^{-1}+1 \otimes y^{\prime}$ и $y^{\prime} x=x y^{\prime}+\gamma q^{-2}\left(K^{-1}-K\right)$. Можно считать, что $-\gamma q^{-2}$ равно 0 или 1 .

\section{СПИСОК ЦИТИРОВАННОЙ ЛИТЕРАТУРЫ}

[1] Beattie M. An isomorphism theorem for Ore extension Hopf algebras // Comm. Algebra. 2000. № 2. P. 569-584.

[2] Beattie M., Dascalescu S., Grunenfelder L. Constructing pointed Hopf algebras by Ore extensions // Algebra. 2000. V. 225. P. 743-770.

[3] Delvaux L. Pairing and Drinfel'd-double of Ore extensions // Comm. Algebras. 2001. V. 29. № 7. P. 3167-3177.

[4] Nenciu A. Cleft extensions for a class of pointed Hopf algebras constructed by Ore extensions // Comm. Algebra. 2001. V. 29. № 5. P. 1959-1981.

[5] Nenciu A. Quasitriangular structures for a class of pointed Hopf algebras constructed by Ore extensions // Comm. Algebra. 2001. V. 29. № 8. P. 3419-3432.

[6] McConnel J.C., Robson J.C. Noncommutative Noetherian rings. New-York: Wiley-Interscience, 1987.

[7] Montgomery S. Hopf algebras and their actions on rings // Reg. Conf. Series in Math. 1993. V. 82.

[8] Диксмье ЖК. Универсальные обертьвающие алгебры. М.: Мир, 1978. 